Piezoelectric composite oscillator for measuring mechanical spectroscopy in small samples that non-match in half wavelength

This content has been downloaded from IOPscience. Please scroll down to see the full text.

2016 Meas. Sci. Technol. 27035902

(http://iopscience.iop.org/0957-0233/27/3/035902)

View the table of contents for this issue, or go to the journal homepage for more

Download details:

IP Address: 130.206.166.249

This content was downloaded on 19/01/2016 at 21:00

Please note that terms and conditions apply. 


\title{
Piezoelectric composite oscillator for measuring mechanical spectroscopy in small samples that non-match in half wavelength
}

\author{
F G Bonifacich ${ }^{1}$, O A Lambri ${ }^{1}$, J I Pérez Landazábal ${ }^{2,3}$, V Recarte ${ }^{2,3}$, \\ G I Zelada ${ }^{1}$, R R Mocellini ${ }^{1}$, V Sánchez Alarcos ${ }^{2}$, A Marenzana ${ }^{4,6}$ \\ and F Plazaola ${ }^{5}$ \\ ${ }^{1}$ Laboratorio de Materiales, Escuela de Ingeniería Eléctrica, Centro de Tecnología e Investigación \\ Eléctrica (CETIE), Facultad de Ciencias Exactas, Ingeniería y Agrimensura, Universidad Nacional de \\ Rosario-CONICET, Avda. Pellegrini 250, (2000) Rosario, Argentina \\ 2 Departamento de Física, Universidad Pública de Navarra, Campus de Arrosadía 31006 Pamplona, Spain \\ ${ }^{3}$ Institute for Advanced Materials (INAMAT), Universidad Pública de Navarra, Campus de Arrosadía \\ 31006 Pamplona, Spain \\ ${ }^{4}$ Instituto de Física Rosario-CONICET, Avda. 27 de Febrero 210 bis, 2000 Rosario, Argentina \\ 5 Elektrika eta Elektronika Saila, Zientzia eta Teknologia Fakultatea, Euskal Herriko Unibertsitatea, P.K. \\ 644, 48080 Bilbao, Spain
}

E-mail: olambri@fceia.unr.edu.ar

Received 11 September 2015, revised 7 December 2015

Accepted for publication 9 December 2015

Published 19 January 2016

\begin{abstract}
A novel piezoelectric device for measuring mechanical spectroscopy as a function of temperature and strain has been developed. The new equipment involves five oscillating elements, a crystal driver, two spacer bars, the sample and the crystal gauge. The spacer bars and the sample do not match in frequency. The device developed here results in an important solution for measuring mechanical spectroscopy in small samples, where the condition of match in frequency cannot be satisfied. Mechanical spectroscopy measurements were performed in free decay with the equipment working in an out of tune condition. The associated mathematical equations required for the measurement process have also been developed. In addition, the new equipment was successfully used for the measurement of different types of materials: metals, polymers and ferromagnetic shape memory alloys.
\end{abstract}

Keywords: mechanical spectroscopy, composite piezoelectric oscillator, short and brittle samples, unmatched samples, ferromagnetic shape memory alloys

(Some figures may appear in colour only in the online journal)

\section{Introduction}

Mechanical spectroscopy (MS), referred to as the internal friction method in the early literature, involves the simultaneous measurement of damping and the elastic modulus as a function of temperature, strain or frequency. This technique is

${ }^{6}$ Passed away. very sensitive to the microstructural state of the sample and it has been widely applied for the characterization of materials. In fact, MS offers unique opportunities for studying interaction processes between defects in materials [1].

Amongst all the experimental techniques used for measuring MS, the piezoelectric method stands out, and is widely applied in physical acoustic and higher-order phase transition studies [1-3]. 


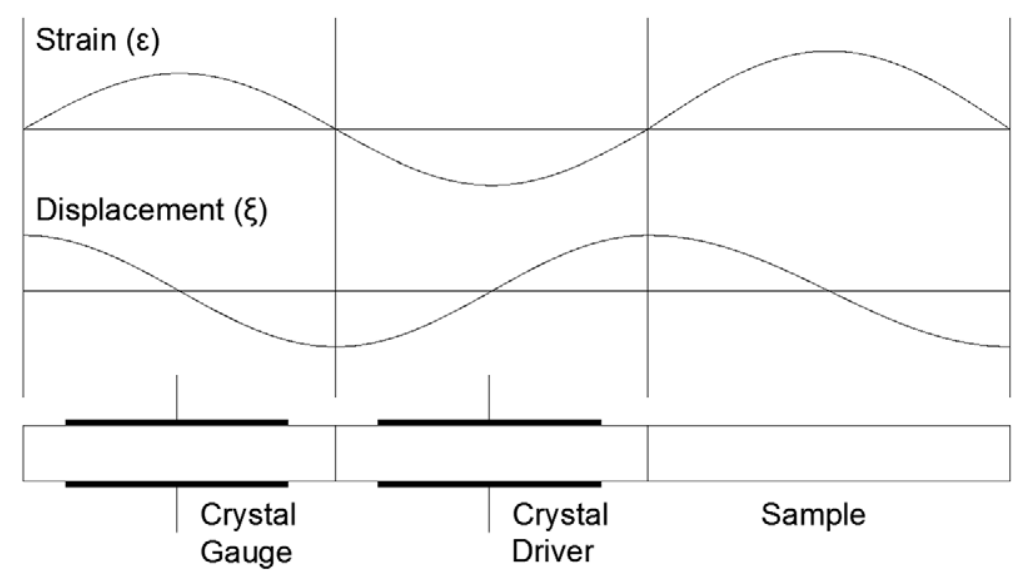

Figure 1. Arrangement for the Marx three-component oscillator. All elements are matched in frequency, and displacement is at a maximum at the interface and the strain is continuous.

In the piezoelectric method, first introduced by Quimby [4], the specimen is cemented to a quartz crystal, and the application of a potential difference across the quartz crystal causes the two to vibrate as a single resonant body. The method was improved by Balamuth [5], who matched the fundamental frequency of the quartz to that of the specimen. In addition, Cooke [6] and Michener and Handloser [7] have discussed methods of evaluating data and experimental setups, respectively. A three-component piezoelectric resonator was introduced by Marx [8]. In this method, an additional quartz crystal (the gauge), which is also matched in frequency, is attached to the free end of the first crystal. This gives a signal that measures the vibrational strain amplitude of the specimen. The ratio of the driving e.m.f. to the gauge signal resonance is proportional to the damping value [9]. Robinson and Edgar [10] carried out a detailed study of a three-element piezoelectric oscillator by considering its equivalent electrical circuit. A different arrangement of crystals and sample was used by Harmouche and Wolfenden [11] and Povolo et al [12], who cemented the gauge between the driver and the sample. They included also a fourth element, the so-called spacer or dummy bar. The furnace and the sample were placed at the bottom of the experimental device, while the crystals were at the top. This configuration is the opposite to the one used by Marx and Sivertsen [13] for performing piezoelectric measurements as a function of temperature, in which crystals were placed at the bottom.

Devine et al [14], Robinson et al [15] and Devine and Robinson [16] introduced a specimen sandwiched between two fused quartz rods in order to form a new one-half wavelength component. In fact, this configuration involves a driver, a gauge, and a third component that consists of a sample sandwiched between fused quartz rods. The whole sandwich exhibits a multiple of a half wavelength. Thus, samples that do not fit into multiples of half wavelength can be measured at room temperature in a resonant tuned device.

In the present work, a novel piezoelectric equipment, and its related mathematical equations, for measuring MS in small samples that do not match in frequency are shown. The new device is a five-component oscillator, where three of the components are unmatched in frequency. The equipment allows us to perform measurements as a function of temperature and strain for low and high damping materials. In addition, the device does not measure during the tuning in frequency, but in free decaying oscillations.

It should be noted that the equipment developed here is an important solution for measuring small samples, often involved in the pilot development of new alloys, that cannot be matched in frequency. However, it is not restricted to this case only. In addition, the new device could also be an important solution for measuring mechanical spectroscopy in brittle samples.

MS measurements as a function of temperature and strain were performed using the new equipment in polycrystalline niobium to check that the equipment functioned well. In addition, the device was successfully used to determine the martensitic transition temperature in small and brittle samples of $\mathrm{Ni}-\mathrm{Mn}-\mathrm{Sn}$ ferromagnetic shape memory alloys [17-20].

\section{Theoretical background}

The Marx three-component piezoelectric resonator, figure 1, requires that the lengths of the components are frequencymatched to their half wavelengths. In this way, both the displacement and the strain waves exhibit continuous behaviour through the composite oscillator. The condition of zero strain and maximum displacement is satisfied at the end of each element. The half wavelength of the displacement wave matches exactly to the length of each element and then the strain wave, which is obtained from the derivative of the displacement, is shifted in $\pi / 2[1,2,8]$. In addition, from the equivalent electrical circuit of the Marx three-component oscillator, the wellknown equations for frequency, $f$, and damping, $Q^{-1}$, can be obtained $[1,10,21]$

$$
\begin{gathered}
f_{\mathrm{T}}^{2} m_{\mathrm{T}}=f_{1}^{2} m_{1}+f_{2}^{2} m_{2}+f_{3}^{2} m_{3} \\
Q_{\mathrm{T}}^{-1} m_{\mathrm{T}}=Q_{1}^{-1} m_{1}+Q_{2}^{-1} m_{2}+Q_{3}^{-1} m_{3}
\end{gathered}
$$

where, $m_{\mathrm{T}}, f_{\mathrm{T}}$ and $Q_{\mathrm{T}}^{-1}$ are the total mass $\left(\Sigma m_{i}\right)$, the oscillating frequency (resonant) and the damping of the whole oscillator, respectively. $m_{i}, f_{i}$ and $Q_{\mathrm{i}}^{-1}$ are the mass, frequency and damping corresponding to the element ' $i$ ', respectively. 

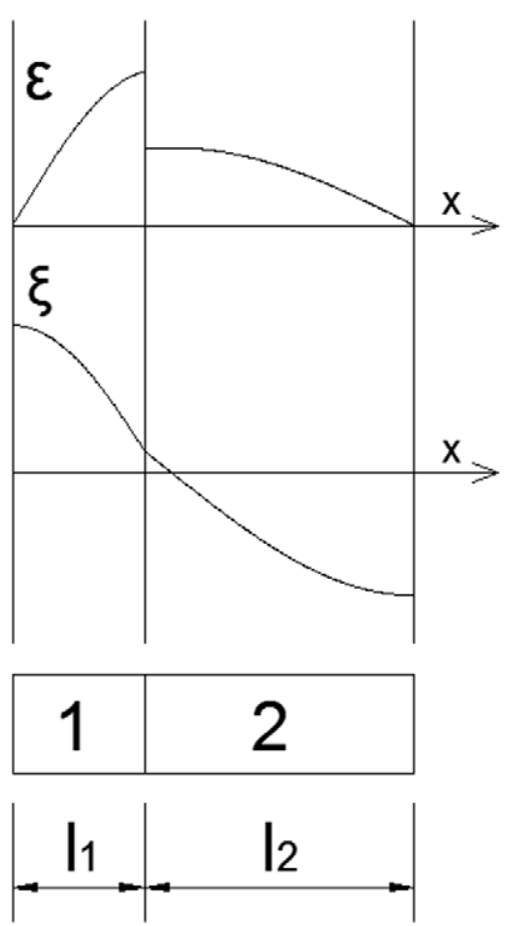

Figure 2. Arrangement for the two-component oscillator (1 and 2) with their lengths non-matched in frequency. The displacement $(\xi)$ exhibits a peak shape at the interface and the strain $(\varepsilon)$ is not continuous.

$i=1-3$, correspond to crystal gauge, crystal driver and sample, respectively (see figure 1). As can be easily inferred from equations (1) and (2) the squared frequency and damping of the whole oscillator are averaged values weighted by the total mass of their components $[1,2,8,10]$.

Let us now consider an oscillator configuration involving two cemented elements, 1 and 2, whose lengths $\left(l_{1}\right.$ and $\left.l_{2}\right)$ are unmatched in half wavelength, as shown in figure 2. Both elements are excited by an external sinusoidal force, giving rise to oscillatory behaviour. Nevertheless, as a consequence of the non-match in half wavelength, the displacement is continuous due to the boundary condition of the cemented area between elements 1 and 2, but it does not exhibit single sinusoidal behaviour at the cemented area (the interface between elements 1 and 2). Thus, the strain wavelength is not continuous through the composite oscillator. In addition, each element of the oscillator does not have the same resonance frequency. Therefore, the frequency and damping of the composite oscillator cannot be directly related to those of each element with equations (1) and (2).

In order to find the relationships in frequency and damping between the composite oscillator from figure 2 and each component element, the following analysis must be carried out. Firstly, let us establish the equations for the frequency and subsequently the corresponding ones for the damping.

Obtaining a displacement with a period of $\pi$ requires that the number of wavelengths must be an integer number of half wavelengths, then for the arrangement shown in figure 2 the following equation can be written [14]

$$
\frac{l_{1}}{\lambda_{1} / 2}+\frac{l_{2}}{\lambda_{2} / 2}=n
$$

where $l_{1}, \lambda_{1}$ and $l_{2}, \lambda_{2}$ are the length and wavelength of elements 1 and 2, respectively, and $\mathrm{n}$ is an integer number that, for this case, equals 1 . Thus, by setting the frequency of the driver to the resonance, and knowing the speed of sound in one of the components, the wavelength of the other can be obtained.

On the other hand, the damping relationships for the oscillator arrangement shown in figure 2 can be obtained from the definition of damping as the ratio between the energy loss by irreversibility and the stored energy, that is $[1,16,22]$

$Q_{\mathrm{T}}^{-1}=\frac{1}{2 \pi} \frac{\Delta W_{\mathrm{T}}}{W_{\mathrm{T}}}=\frac{1}{2 \pi} \frac{\Delta W_{1}+\Delta W_{2}}{W_{1}+W_{2}}=\frac{Q_{1}^{-1} W_{1}+Q_{2}^{-1} W_{2}}{W_{1}+W_{2}}$

and then

$$
Q_{\mathrm{T}}^{-1} W_{\mathrm{T}}=Q_{1}^{-1} W_{1}+Q_{2}^{-1} W_{2}
$$

where $\Delta W_{\mathrm{T}}, \Delta W_{1}$, and $\Delta W_{2}$ are the loss energy by irreversibility for the whole oscillator, and the elements 1 and 2, respectively. $W_{\mathrm{T}}, W_{1}$, and $W_{2}$ are the stored energy in the cycle (at the peak of the cycle) for the whole oscillator, and the elements 1 and 2, respectively [1, 9, 22, 23].

Obtaining the storage energies involves the product between the stress and strain, so, the following paragraphs are devoted to determining the spatial dependence of the oscillating strain in each element. The strain at any point in each element, $\varepsilon_{i}(x)$, with $i=1,2$ takes the form

$$
\varepsilon_{i}(x)=\varepsilon_{m i} \sin \left(\frac{2 \pi}{\lambda_{i}} x-c_{i}\right)
$$

where $x$ is the position of the element from the origin, and $\varepsilon_{m i}$, $\lambda_{i}$ and $c_{i}$ are the maximum oscillating strain, the wavelength and the phase angle, respectively, for the component element ' $i$ ', with $i=1,2$. Then, for the two-element composite oscillator from figure 2 , the stored energies can be written as (with $i=1,2$ )

$$
W_{i}=\frac{1}{2} A_{i} E_{i} \varepsilon_{m i}^{2} \int_{l_{i-1}}^{l_{i-1}+l_{i}} \sin ^{2}\left(\frac{2 \pi}{\lambda_{i}} x-c_{i}\right) \mathrm{d} x
$$

where $A_{i}$ and $E_{i}$ are the area and the elastic modulus for the element ' $i$ ', respectively. $l_{0}=0$, which results for the case where ' $i$ ' $=1$ is used for the integration of the first interval.

For element number 1 in figure $2 c_{1}=0$, then we can write

$$
W_{1}=\frac{1}{2} A_{1} E_{1} \varepsilon_{m 1}^{2}\left[\frac{l_{1}}{2}-\frac{\lambda_{1}}{4 \pi} \sin \left(\frac{4 \pi}{\lambda_{1}} l_{1}\right)\right]
$$

while, for element number 2 in figure 2, we have

$$
\begin{aligned}
W_{2} & =\frac{1}{2} A_{2} E_{2} \varepsilon_{m 2}^{2} \int_{l_{1}}^{l_{1}+l_{2}} \sin ^{2}\left(\frac{2 \pi}{\lambda_{2}} x-c_{2}\right) \mathrm{d} x \\
& =\frac{1}{2} A_{2} E_{2} \varepsilon_{m 2}^{2}\left[\frac{l_{2}}{2}-\frac{\lambda_{2}}{4 \pi}\left[\sin \left(\frac{4 \pi}{\lambda_{2}}\left(l_{1}+l_{2}\right)-2 c_{2}\right)-\sin \left(\frac{4 \pi}{\lambda_{2}} l_{1}-2 c_{2}\right)\right]\right]
\end{aligned}
$$

where $c_{2}$ can be obtained from the boundary condition at the point $x=l_{1}+l_{2}$. In fact, considering that $c_{2}$ is the initial out of phase in the element \#2, it can be easily obtained that 


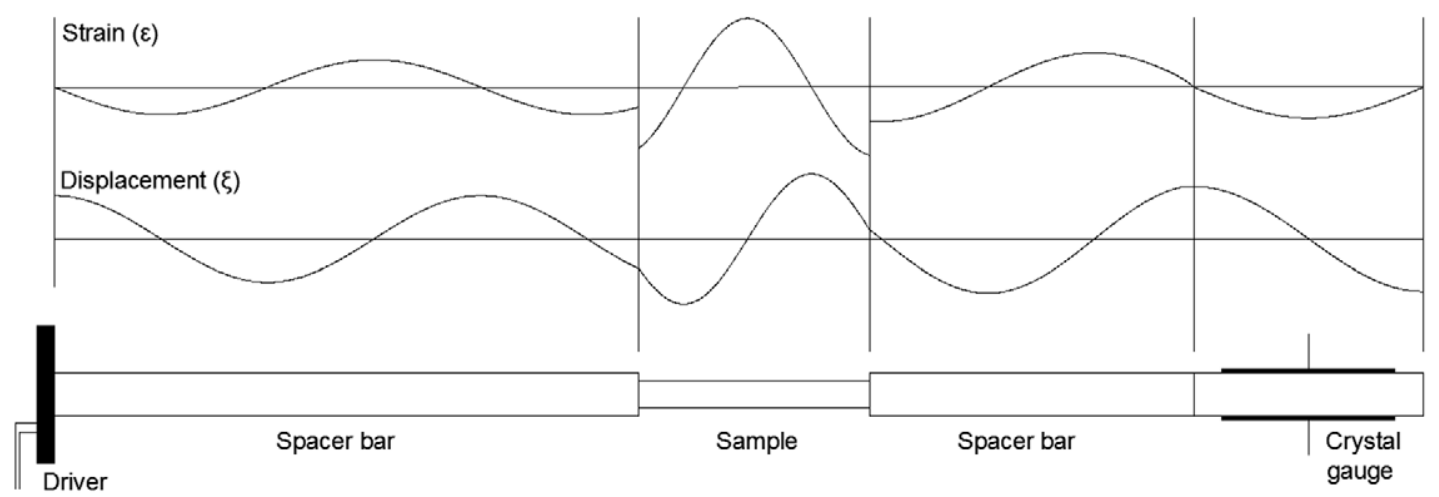

Figure 3. A novel five-component oscillator. The driver is a thin-flat power quartz crystal. The two spacer bars and the sample do not match in frequency. The crystal gauge is cut to match in frequency. The displacement wave, even when continuous, exhibits peak shape behaviour at the interface and the strain is not continuous.

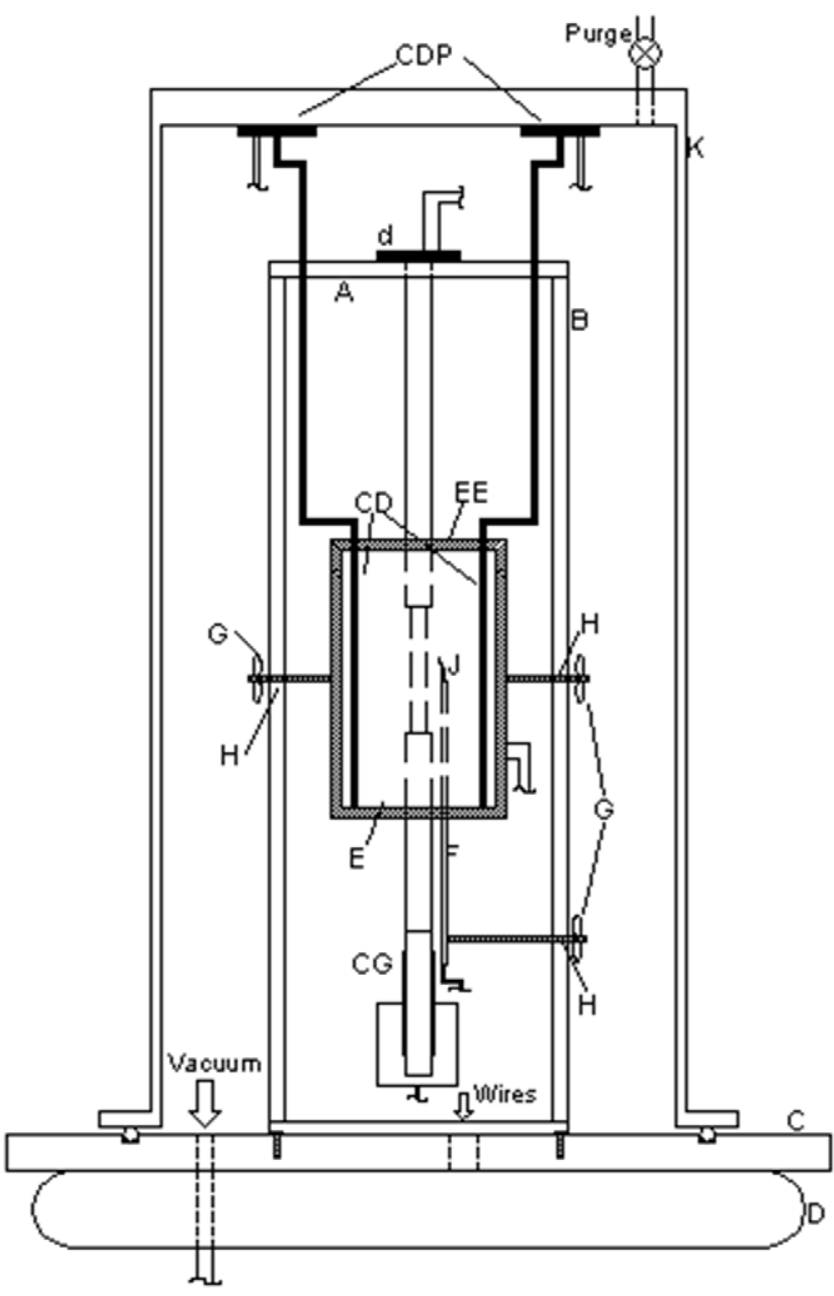

Figure 4. A schematic representation of the new piezoelectric device for measuring mechanical spectroscopy as a function of temperature and strain. See labels in the text.

$$
c_{2}=\frac{2 \pi}{\lambda_{2}}\left(l_{1}+l_{2}\right)-\pi
$$

Finally, in order to calculate equations (8) and (9), the values of strains $\varepsilon_{m 1}$ and $\varepsilon_{m 2}$ should be obtained. From the boundary condition of equilibrium of forces at the interface between elements number 1 and number 2 , a relation between the maximum strains of each element can be derived, such that

$$
\varepsilon_{m 1}=\varepsilon_{m 2} \frac{E_{2} A_{2} \sin \left(\frac{2 \pi}{\lambda_{2}} l_{2}\right)}{E_{1} A_{1} \sin \left(\frac{2 \pi}{\lambda_{1}} l_{1}\right)}
$$

and from there, one of them can be easily obtained as a function of the other one.

\section{Mathematical expressions governing the novel five-element composite oscillator with three unmatched components}

Let us consider in this section a composite oscillator involving a thin-flat driver, two spacer bars, a sample and crystal gauge assembled as shown in figure 3 . The spacer bars and sample are non-matched in half wavelength. The driver is a thin-flat piezoelectric quartz crystal. By contrast, the gauge is a typical quartz crystal cut at half wavelength. The length and mass of the driver are not significant in comparison to the other elements so they can be neglected. Therefore, the driver will be assumed to be an elastic membrane that generates a plane wave front. This assumption allows us to consider the displacement and strain, maximum and nil, respectively, at the interface.

As previously noted, the displacement, at the interface between elements in a composite oscillator involving frequency unmatched components, does not exhibit single sinusoidal behaviour, see figure 2. Thus, the strain wavelength will not be continuous throughout the composite oscillator. In addition, each element of the oscillator does not have the same resonance frequency. Nevertheless, the resonance frequency is given by equation (12). From a straightforward analysis, the modified form of equation (3) for the five-element composite oscillator can be written as

$$
\frac{l_{\mathrm{sb} 1}}{\lambda_{\mathrm{sb} 1} / 2}+\frac{l_{\mathrm{S}}}{\lambda_{\mathrm{s}} / 2}+\frac{l_{\mathrm{sb} 2}}{\lambda_{\mathrm{sb} 2} / 2}+\frac{l_{\mathrm{CG}}}{\lambda_{\mathrm{CG}} / 2}=n
$$

where the sub-indexes $s_{b i}$ (with $i=1,2$ ) are related to the spacer bars, $\mathrm{S}$ corresponds to the sample, $\mathrm{CG}$ is for the crystal gauge and $n$ is an integer, which for this case equals 7 (see 
figure 3). Spacer bars 1 and 2 will be labelled, hereafter, to denote left and right in figure 3.

Thus, by assuming that the speed of sound in the spacer bars (for instance quartz or Pyrex) and the crystal gauge are known, the wavelength corresponding to the sample can be easily determined.

Regarding the calculus of the stored energy in the spacer bars and in the sample (see figure 3), we can obtain firstly the phase-constants, $c_{i}$, and subsequently $\varepsilon_{m i}$.

However, for clarity, for the five-component composite oscillator, the phase constants will be obtained from the displacements expressions. Strain expressions can be deduced easily from the derivative of the corresponding displacement. Thus, by considering the origin of the coordinates system at the left end of the composite oscillator, the expressions for the displacement in each spacer bar and in the sample result in

$$
\begin{gathered}
\xi_{\mathrm{sb} 1}(x)=\xi_{\mathrm{sb} 1 \mathrm{Max}} \cos \left(\frac{2 \pi}{\lambda_{\mathrm{sb} 1}} x\right) \\
\xi_{\mathrm{S}}(x)=\xi_{\mathrm{SMax}} \cos \left(\frac{2 \pi}{\lambda_{\mathrm{s}}} x-c_{2}\right) \\
\xi_{\mathrm{sb} 2}(x)=\xi_{\mathrm{sb} 2 \mathrm{Max}} \cos \left(\frac{2 \pi}{\lambda_{\mathrm{sb} 1}} x-c_{3}\right) .
\end{gathered}
$$

To obtain $c_{3}$, we consider that the displacement at the interface between spacer bar 2 and the crystal gauge is at a maximum. Then, from equation (15) evaluated at the interface with the crystal gauge, we can write

$$
\begin{aligned}
& \xi_{\mathrm{sb} 2}\left(l_{\mathrm{sb} 1}+l_{\mathrm{S}}+l_{\mathrm{sb} 2}\right)=\xi_{\mathrm{sb} 2 \mathrm{Max}} \cos \left(\frac{2 \pi}{\lambda_{\mathrm{sb}}}\left(l_{\mathrm{sb} 1}+l_{\mathrm{S}}+l_{\mathrm{sb} 2}\right)-c_{3}\right) \\
& =\xi_{\mathrm{sb} 2 \mathrm{Max}}
\end{aligned}
$$

and $c_{3}$ is given by

$$
c_{3}=\frac{2 \pi}{\lambda_{\mathrm{sb}}}\left(l_{\mathrm{sb} 1}+l_{\mathrm{S}}+l_{\mathrm{sb} 2}\right)
$$

To obtain $c_{2}$, the boundary conditions for the displacement and the force at the interface between spacer bar 1 and the sample are considered. Indeed, both the displacement and the force, perpendicular to the cross section, must be the same in each element (see figure 3). Then, for the displacement it holds that

$$
\xi_{\mathrm{sb} 1}\left(l_{\mathrm{sb} 1}\right)=\xi_{\mathrm{S}}\left(l_{\mathrm{sb} 1}\right)
$$

thus

$$
\xi_{\mathrm{sb} 1 \mathrm{Max}} \cos \left(\frac{2 \pi}{\lambda_{\mathrm{sb}}} l_{\mathrm{sb} 1}\right)=\xi_{\mathrm{SMax}} \cos \left(\frac{2 \pi}{\lambda_{\mathrm{S}}} l_{\mathrm{sb} 1}-c_{2}\right)
$$

while for the force,

$$
F_{\mathrm{sb} 1}=F_{\mathrm{S}}
$$

and

$$
E_{\mathrm{sb}} A_{\mathrm{sb}} \varepsilon_{\mathrm{sb} 1}\left(l_{\mathrm{sb} 1}\right)=E_{\mathrm{S}} A_{\mathrm{S}} \varepsilon_{\mathrm{S}}\left(l_{\mathrm{sb} 1}\right)
$$

$$
\begin{aligned}
& E_{\mathrm{sb}} A_{\mathrm{sb}} \frac{2 \pi}{\lambda_{\mathrm{sb}}} \xi_{\mathrm{sb} 1 \mathrm{Max}} \sin \left(\frac{2 \pi}{\lambda_{\mathrm{sb}}} l_{\mathrm{sb} 1}\right) \\
& =E_{\mathrm{S}} A_{\mathrm{S}} \frac{2 \pi}{\lambda_{\mathrm{S}}} \xi_{\mathrm{SMax}} \sin \left(\frac{2 \pi}{\lambda_{\mathrm{S}}} l_{\mathrm{sb} 1}-c_{2}\right) .
\end{aligned}
$$

By working mathematically we obtain

$$
E_{\mathrm{sb}} A_{\mathrm{sb}} \frac{2 \pi}{\lambda_{\mathrm{sb}}} \operatorname{tg}\left(\frac{2 \pi}{\lambda_{\mathrm{sb}}} l_{\mathrm{sb} 1}\right)=E_{\mathrm{S}} A_{\mathrm{S}} \frac{2 \pi}{\lambda_{\mathrm{S}}} \operatorname{tg}\left(\frac{2 \pi}{\lambda_{\mathrm{S}}} l_{\mathrm{sb} 1}-c_{2}\right)
$$

from which $c_{2}$ takes the form,

$$
c_{2}=\frac{2 \pi}{\lambda_{\mathrm{S}}} l_{\mathrm{sb} 1}-\operatorname{tg}^{-1}\left(\frac{E_{\mathrm{sb}} A_{\mathrm{sb}} \lambda_{\mathrm{S}}}{E_{\mathrm{S}} A_{\mathrm{S}} \lambda_{\mathrm{sb}}} \operatorname{tg}\left(\frac{2 \pi}{\lambda_{\mathrm{sb}}} l_{\mathrm{sb} 1}\right)\right) .
$$

Let us now obtain the $\varepsilon_{m i}$ terms. As noted above, the displacement at the interface of the two adjacent elements of the composite oscillator must be the same. Then, we can write for spacer bar 1 and the sample

$$
\xi_{\mathrm{sb} 1}\left(l_{\mathrm{sb} 1}\right)=\xi_{\mathrm{S}}\left(l_{\mathrm{sb} 1}\right) .
$$

By taking the derivative, we can write

$\varepsilon_{\mathrm{sb} 1 \mathrm{Max}} \frac{\lambda_{\mathrm{sb}}}{2 \pi} \cos \left(\frac{2 \pi}{\lambda_{\mathrm{sb}}} l_{\mathrm{sb} 1}\right)=\varepsilon_{\mathrm{SMax}} \frac{\lambda_{\mathrm{S}}}{2 \pi} \cos \left(\frac{2 \pi}{\lambda_{\mathrm{S}}} l_{\mathrm{sb} 1}-c_{2}\right)$.

A rearrangement of equation (26) leads to

$$
\frac{\varepsilon_{\text {sb1Max }}}{\varepsilon_{\mathrm{SMax}}}=\frac{\lambda_{\mathrm{S}}}{\lambda_{\mathrm{sb}}} \frac{\cos \left(\frac{2 \pi}{\lambda_{\mathrm{S}}} l_{\mathrm{sb} 1}-c_{2}\right)}{\cos \left(\frac{2 \pi}{\lambda_{\mathrm{sb}}} l_{\mathrm{sb} 1}\right)} .
$$

Similarly, for the interface between spacer bar 2 and the sample, we can obtain

$$
\frac{\varepsilon_{\mathrm{sb} 2 \mathrm{Max}}}{\varepsilon_{\mathrm{SMax}}}=\frac{\lambda_{\mathrm{S}}}{\lambda_{\mathrm{sb}}} \frac{\cos \left(\frac{2 \pi}{\lambda_{\mathrm{S}}}\left(l_{\mathrm{sb} 1}+l_{\mathrm{S}}\right)-c_{2}\right)}{\cos \left(\frac{2 \pi}{\lambda_{\mathrm{sb}}}\left(l_{\mathrm{sb} 1}+l_{\mathrm{S}}\right)-c_{3}\right)} .
$$

Finally, the maximum strain in spacer bar 2 can be obtained from the continuity condition of the strains between the crystal gauge (CG) and the bar, that is

$$
\varepsilon_{\mathrm{sb} 2 \mathrm{Max}}=\varepsilon_{\mathrm{CGMax}} \frac{\lambda_{\mathrm{CG}}}{\lambda_{\mathrm{sb} 2}} .
$$

The maximum strain in the crystal gauge $\varepsilon_{\mathrm{CG} \text { Max }}$ is given by the well-known expression [8]

$$
\varepsilon_{\mathrm{CGMax}}=V_{\mathrm{e}} \frac{1}{f_{\mathrm{c}} l_{\mathrm{c}}} \sqrt{\frac{1}{2 m_{\mathrm{c}} f_{\mathrm{c}} R F_{\mathrm{c}}}}=\beta V_{\mathrm{g}}
$$

where $V_{\mathrm{e}}$ is the excitation voltage, $f_{\mathrm{c}}$ is the resonant frequency, $F_{\mathrm{c}}$ is the damping of the oscillator and $l_{\mathrm{c}}, m_{\mathrm{c}}$ and $R$ are the length and mass of the crystal and the resistance of the equivalent circuit, respectively. However, the expression for the maximum strain reported in [10] can also be used, that is

$$
\varepsilon_{\mathrm{CGMax}}=\frac{28.1}{\lambda_{\mathrm{CG}} l_{\mathrm{CG}}} C_{m} V_{\mathrm{g}}
$$



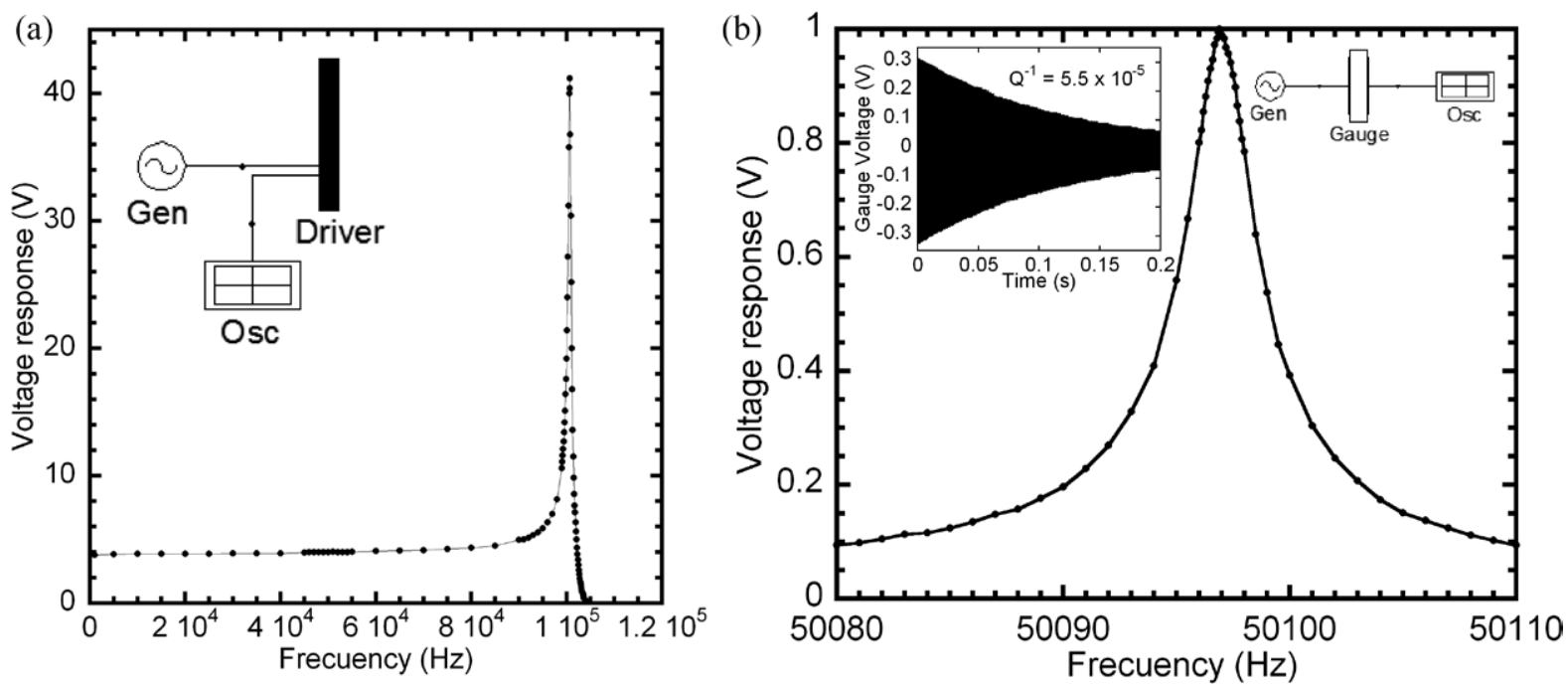

Figure 5. (a) Response in frequency for the flat power quartz driver. Inset: circuit used for the driver response measurement. (b). Response in frequency for the crystal gauge. Inset: left, free decay of the gauge; right, circuit used for the measurement.

where $C_{m}$ is an additional capacitance placed across the crystal gauge. By using equation (30) or (31) in the calculations, clear differences could not be found. Therefore, as equation (30) is widely known, it will be used in the present work.

By knowing $\varepsilon_{\mathrm{CGMax}}$, the strain values of $\varepsilon_{\mathrm{sb} 2 \mathrm{Max}}, \varepsilon_{\mathrm{SMax}}$ and $\varepsilon_{\text {sb1Max }}$ can be obtained through equations (27)-(29). In addition, if the cross section, the modulus and the length of each component of the oscillator are known, the storage energies for the spacer bars $\left(W_{\mathrm{sb} 1}, W_{\mathrm{sb} 2}\right)$, the sample $\left(W_{\mathrm{S}}\right)$ and the crystal gauge $\left(W_{\mathrm{CG}}\right)$ can be calculated from analogous integrals to equation (7).

Consequently, all the expressions needed to obtain the damping response of the sample from the general equation (5) have been developed. By working analogously to equation (5) we can write

$$
Q_{\mathrm{T}}^{-1} W_{\mathrm{T}}=Q_{\mathrm{sb} 1}^{-1} W_{\mathrm{sb} 1}+Q_{\mathrm{S}}^{-1} W_{\mathrm{S}}+Q_{\mathrm{sb} 2}^{-1} W_{\mathrm{sb} 2}+Q_{\mathrm{CG}}^{-1} W_{\mathrm{CG}}
$$

where $W_{\mathrm{T}}$ is the storage energy of the whole oscillator, and $Q_{\mathrm{S}}^{-1}, Q_{\mathrm{CG}}^{-1}, Q_{\mathrm{sb} 1}^{-1}, Q_{\mathrm{sb} 2}^{-1}$ and $Q_{\mathrm{T}}^{-1}$ are the loss factors of the sample, the crystal gauge, the spacer bars (sb1 and sb2) and the total for the whole oscillator, respectively. The method for obtaining $Q^{-1}$ for each component of the oscillator is described in section 5.1. Therefore, if the damping of each component is known, the damping of the sample from equation (32) can be calculated.

\section{Experimental}

\subsection{Five-element composite oscillator device}

Figure 4 shows the schematic representation of the experimental setup of the novel device. The oscillator hangs from the driver, $d$, which is supported (not clamped) by a rubber cantilever fixed to a beam, A, which is housed in a tower, B. The tower is fixed to a fused lead base, C, which is mechanically isolated by means of an air camera, D. The electrical furnace, E, and thermocouple of K-type, F, are fixed to the tower $\mathrm{B}$, by means of variable screws, $\mathrm{G}$, running inside guides, $\mathrm{H}$.
The surface, top and bottom of the furnace are thermally isolated by means of a ceramic blanket, EE, of $20 \mathrm{~mm}$ thickness. The thermocouple is positioned $1 \mathrm{~mm}$ from the sample and it has, at the hot juncture, a cup of copper, J, in order to measure the real temperature of the sample without distortion by the vacuum as was suggested in previous works $[8,11,12]$. The influence of the heat flow from the furnace to the crystal driver and gauge was carefully checked in order to obtain non-significant effects on their frequencies and voltages. The maximum admissible temperature of operation of the equipment is $800 \mathrm{~K}$.

For low temperature measurements the electrical furnace is replaced by a copper rectangular parallelepiped cooler device, $\mathrm{CD}$, which has cemented to it four Peltier plates, CDP. This cooler device allows us to perform measurements from $230 \mathrm{~K}$ up to room temperature (RT). Heating and cooling runs are controlled by means of a PID (Novus N480D) temperature controller.

The chamber, $\mathrm{K}$, allows the equipment to work under a protective atmosphere.

An ultrasonic wave is generated by a thin-flat power commercial piezoelectric quartz driver, produced by Apple Vista Technology Ltd (d in figure 4). It is excited by a synthesized waveform generator, Rigol DG 1022, plus a power amplifier of plane response with low harmonic distortion. The diameter, thickness and weight of the driver are $25 \mathrm{~mm}, 1 \mathrm{~mm}$ and $4.55 \mathrm{~g}$, respectively.

The frequency response of the driver is shown in figure 5(a). The almost flat zone around $50 \mathrm{kHz}$ is used to drive the system, so the driver is exciting the oscillator working outside of its resonance frequency. The frequency response of the driver was measured according to the circuit shown in the inset in figure 5(a).

The crystal gauge (CG in figure 4) is made of a quartz piezoelectric crystal of high quality factor with a parallelepiped shape of $5.00 \times 5.00 \times 56.10 \mathrm{~mm}$ and a weight of $4.10 \mathrm{~g}$, with gold deposited faces and with a characteristic frequency of around $50 \mathrm{kHz}$. This crystal was provided by 
Bliley Electric Company. Once the mechanical excitation is switched off, the free decaying of oscillations from the gauge can be recorded. The behaviour of the frequency of the gauge is shown in figure 5(b). A typical decaying oscillation for the gauge is included in the inset.

In order to assess whether mechanical excitation can be produced by this kind of piezoelectric driver, working out of the resonance condition, the driver was cemented to a Bliley's quartz crystal. A clear mechanical oscillation was corroborated by the gauge in the frequency range $40-60 \mathrm{kHz}$.

The crystal gauge signal was amplified by a $150 \mathrm{kHz}$ amplifier with variable output, see the appendix. The decay was recorded by a high-speed digital storage oscilloscope, Rigol DS 1052 E. For data analysis the oscilloscope was connected to a personal computer.

\subsection{Samples, cement and spacer bars}

Samples used in this work were pure niobium and ferromagnetic shape memory alloys (FSMA).

The high-purity polycrystalline niobium samples were two rods of $2 \mathrm{~mm}$ diameter and lengths $48.84 \mathrm{~mm}$ and $5.1 \mathrm{~mm}$, called $\mathrm{Nb} 1$ and $\mathrm{Nb} 2$ samples, respectively. Niobium samples were stress-relieved and their oxygen content was around 550 atppm [24-26].

FSMA alloys of composition $\mathrm{Ni}_{50} \mathrm{Mn}_{37} \mathrm{Sn}_{13}$, and $\mathrm{Ni}_{50} \mathrm{Mn}_{37} \mathrm{Sn}_{11} \mathrm{Ti}_{2}$, called hereafter $\mathrm{Ti}=0$ and $\mathrm{Ti}=2$ samples, respectively, were in parallelepiped shape. The dimensions for $\mathrm{Ti}=0$ and $\mathrm{Ti}=2$ were $6.82 \times 8.12 \times 5.34$ and $4.82 \times 5.74 \times 1.5 \mathrm{~mm}$, respectively. It should be noted that the size of the samples is far from a match in frequency.

Polycrystalline FSMA ingots were prepared from high purity elements by arc melting under protective Ar atmosphere. The ingots were homogenized in vacuum quartz ampoules at $1273 \mathrm{~K}$ for $2 \mathrm{~h}$. After homogenization, samples were annealed at $1173 \mathrm{~K}$ in ampoules under Ar for $30 \mathrm{~min}$, followed by quenching in iced water.

Samples were cemented to the oscillator components by using a mixture of sodium silicate and high alumina cement in a proportion of 25/75 in volume. After the cementing process, the entire composite oscillator was placed into the vacuum chamber in order to dry the cemented parts. The system was evacuated in a vacuum better than 90 mTorr for $72 \mathrm{~h}$ at RT. Otherwise, some contribution of the curing process can be recorded during the tests, which masks the true dynamical response of the material under study.

The spacer bars were cylinders of fused quartz of $6 \mathrm{~mm}$ diameter and $157 \mathrm{~mm}$ and $132 \mathrm{~mm}$ length for sb1 and $\mathrm{sb} 2$, respectively. Pyrex bars were also used.

During the cooling and heating runs, the driver frequency at each temperature was changed until the system vibrated at maximum amplitude (highest voltage at crystal gauge). Subsequently, after the system was at maximum strain, the voltage of the driver was changed at will in order to obtain a voltage in the gauge that corresponded to the oscillating strain chosen for the sample during the measurement. As was shown in section 3, this was done using coupling equations (28)-(30). Then, at each temperature, when the driver was switched-off, the free decaying oscillation as a function of time was recorded by means of the digital oscilloscope.

The frequency during the free decay of oscillations of the composite oscillator, is calculated from the recorded decaying oscillation. In addition, the frequency $f$ and wavelength $\lambda$ for each one of the components satisfy the well-known relationship, $f \lambda=v, v$ being the speed of sound in each component. Thus, as was shown in section 3.2, by knowing the speed of sound in the spacer bars and crystal gauge, the change in the wavelength for the sample can be obtained from equation (12). Subsequently, the elastic modulus, $E$, can be calculated for a sample from the usual equation involving the density, $\rho: \rho v^{2}=E$.

For the damping measurement the usual expression for calculating the damping from the slope of the straight line that results from the least squares fitting of the natural logarithm of the decaying areas versus time is used $[1,23]$

$$
\ln \left(A_{m}\right)=\ln \left(A_{0}\right)-\pi Q_{\mathrm{T}}^{-1} m
$$

where $A_{m}$ is the area of the $m$ th decaying oscillation, $A_{0}$ is the initial area of the starting decay oscillation and $m$ is the period number. For damping measurements the same initial and end values of the decay areas were used for eliminating some possible distortion due to the appearance of amplitude dependent damping effects [23]. Once $Q_{\mathrm{T}}^{-1}$ was measured, by coupling equations (13)-(32), the damping of the sample could be calculated. In addition, as a consequence of measuring in free decay mode, the amplitude dependent damping (ADD) behaviour promoted by the appearance of non-linear effects (the doubling of stress does not lead to the doubling of strain) can be easily measured. The damping as a function of the maximum strain on the sample, $\varepsilon_{0}$, was calculated from equation (34) [23, 27-29]

$$
Q_{\mathrm{T}}^{-1}\left(\varepsilon_{0}\right)=-\frac{1}{\pi} \frac{\mathrm{d}\left(\ln \left(A_{m}\right)\right)}{\mathrm{d} m} .
$$

The decay of the oscillations was carried out at constant temperature $(T \pm 0.5 \mathrm{~K})$. Polynomials were fitted to the curve of the decaying areas of the longitudinal vibrations as a function of the period number by means of chi-square fitting. Subsequently equation (34) was applied. Polynomials of degree higher than 1 indicate that $Q_{\mathrm{T}}^{-1}$ is a function of $\varepsilon_{0}$, leading to the appearance of ADD effects, as can be inferred easily. This procedure allows us to obtain the damping as a function of the maximum strain $\left(\varepsilon_{0}\right)$ from free decaying oscillations [23, 27-29]. By coupling equations (13)-(32), the damping of the sample can be obtained at each maximum strain for the whole temperature range of the test. In addition, the strength of ADD effects can be measured through the $S$ parameter, such that [23, 27-29]

$$
S=\frac{\Delta Q_{\mathrm{S}}^{-1}}{\Delta \varepsilon_{0}} .
$$

\section{Experimental results}

\subsection{Reference relaxation process}

In order to check both the behaviour of the equipment and the mathematical work developed above, measurements using 


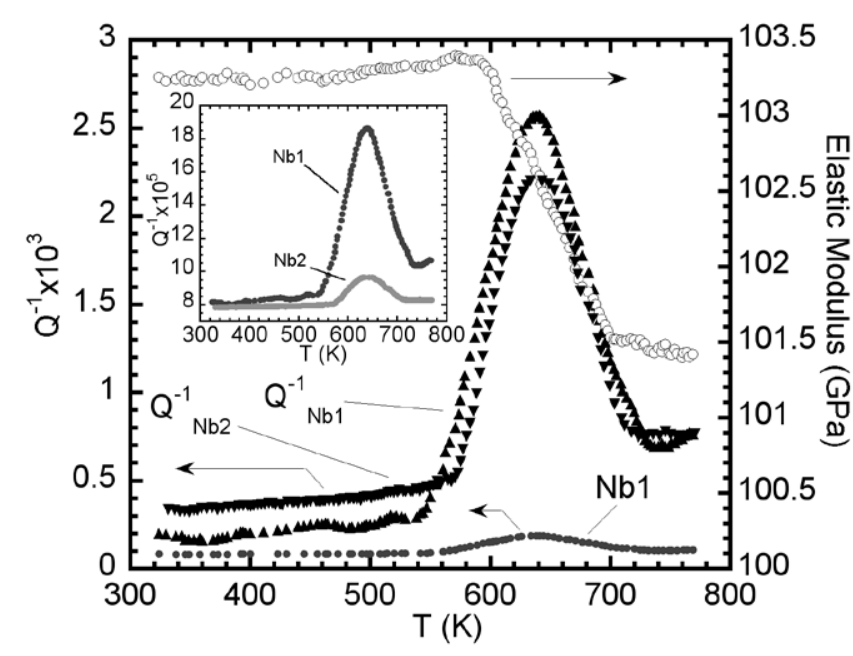

Figure 6. Snoek $\mathrm{Nb}-\mathrm{O}$ peak measured during heating for the $\mathrm{Nb} 1$ and $\mathrm{Nb} 2$ samples. The modulus relaxation for the $\mathrm{Nb} 1$ sample is also shown. $Q_{\mathrm{Nb} 1}^{-1}$ : damping for the Nb1 sample after mathematical treatment. $Q_{\mathrm{Nb} 2}^{-1}$ : damping for the $\mathrm{Nb} 2$ sample after mathematical treatment. Inset: damping directly measured for the $\mathrm{Nb} 1$ and $\mathrm{Nb} 2$ samples.

the new equipment were performed using a widely explored relaxation process. Figure 6 shows the Snoek relaxation of oxygen in niobium measured in the kilohertz range using the new device, with fused quartz spacer bars, during heating at $1 \mathrm{~K} \mathrm{~min}{ }^{-1}$, for the $\mathrm{Nb} 1$ and $\mathrm{Nb} 2$ samples. Tests were performed in a pure argon protective atmosphere at normal pressure. The Snoek relaxation of oxygen in niobium is related to the stress-induced ordering phenomenon of interstitial atoms in the octahedral sites in the bcc lattice [1, 24-26].

The directly measured data, $Q_{\mathrm{T}}^{-1}$, and the resulting spectrum after mathematical treatment of the $\mathrm{Nb} 1$ sample, $Q_{\mathrm{Nb} 1}^{-1}$, are plotted using full circles and full triangles, respectively. In addition, the resulting spectrum for the $\mathrm{Nb} 2$ sample after mathematical treatment, $Q_{\mathrm{Nb} 2}^{-1}$, is also plotted using inverted full triangles. The inset in figure 6 shows the directly measured data, $Q_{\mathrm{T}}^{-1}$, for both $\mathrm{Nb} 1$ and $\mathrm{Nb} 2$ samples. Plotted values for damping after mathematical treatment were obtained by applying equation (32).

Also shown in figure 6 is the behaviour of the Young modulus for the Nb1 sample. The error in the Young modulus was estimated to be around $4 \%$, where the main contribution comes from uncertainties both in the length and the weight of the components of the oscillator.

The damping, $Q_{\mathrm{T}}^{-1}$, and storage energy, $W_{\mathrm{T}}$, for the whole oscillator, and the values of damping, storage energy and elastic modulus for the spacer bars, crystal gauge and niobium samples, evaluated at RT and $650 \mathrm{~K}$ (peak temperature, see figure 6) are shown in table 1 . The values of damping and elastic modulus for the crystal gauge were measured using the configuration shown in the inset in figure 5(b). In addition, the damping and elastic modulus for the spacer bars were measured using the configuration of a matched three-component oscillator, i.e. driver, spacer bar and crystal gauge.

As can be seen from figure 6, the peak heights are in reasonable agreement with the solute content of the samples
(550 atppm) [24, 30, 31]. In addition, the peak temperature and the frequency at the peak temperature, for the spectra $Q_{\mathrm{Nb} 1}^{-1}$ and $Q_{\mathrm{Nb} 2}^{-1}$, are in good agreement with an Arrhenius plot for the Snoek relaxation of oxygen in niobium (activation energy, $H=1.1 \mathrm{eV}$, pre-exponential factor, $\left.\tau_{0}=6 \times 10^{-15} \mathrm{~s}[24-26]\right)$.

The comparison of the spectra for the $\mathrm{Nb} 1$ and $\mathrm{Nb} 2$ samples allows us to determine qualitatively the error bandwidth of the experimental procedure both as a function of the length of the sample and the damping value. For small damping values, close to $5 \times 10^{-4}$, the error bandwidth is around $40 \%$, see figure 6. By contrast, for damping values close to $2 \times 10^{-3}$, the error is decreased to around $15 \%$. The main contribution to the error bandwidth is related to uncertainties in the phase angle $\left(c_{i}\right)$ and, consequently, in the strains in each component of the oscillator (see equations (27) and (28)). Nevertheless, it should be stressed that the scatter of the calculated data during heating and cooling runs is around $2 \%$.

In addition, as can be seen from figure 6, the shape of the spectra $Q_{\mathrm{Nb} 1}^{-1}$ and $Q_{\mathrm{Nb} 2}^{-1}$ do not differ appreciably from the directly measured ones. In addition, the peak temperature is the same for the directly measured curve and the spectrum for the $\mathrm{Nb}$ sample, $Q_{\mathrm{Nb} 1}^{-1}$.

By contrast, if Pyrex spacer bars are used, the real and raw spectra can differ appreciably above $373 \mathrm{~K}$, due to the Pyrex bars making an appreciable contribution to the total damping of the equipment [13]. The directly measured damping values with Pyrex bars are around $1 \times 10^{-3}$.

On the other hand, the measured damping values in the whole temperature range resulted in amplitude independence, i.e. the value of the strength of the amplitude dependent effect, $\mathrm{S}$, was nil within the whole temperature range, in agreement with the literature on Nb-O Snoek relaxation [1, 2, 24-27].

\subsection{Determination of the martensitic transition in FSMA samples}

Figure 7 shows the temperature dependence of the damping and frequency for a ferromagnetic shape memory alloy $(\mathrm{NiMnSn}), \mathrm{Ti}=0$, around the martensitic transformation (MT). The damping peak and the square-frequency (proportional to elastic modulus) drop show that the MT is around $340 \mathrm{~K}$. Another relaxation process seems to appear at around $365 \mathrm{~K}$ just above the MT. It is appropriate to mention here that the frequency shows only a trend, and not the real magnitude of frequency change related to the physical effect. In fact, in pilot alloys the speed of sound cannot be easily found as a function of temperature, so an estimation of the elastic modulus cannot be made. However, when the modulus changes as a consequence of the temperature increase, the speed of sound in the sample will change. Therefore, it is necessary to modify the frequency of excitation in order to keep the system oscillating to maximum amplitude. The frequency measurement of the composite oscillator, during heating or cooling, then allows us to describe the changes in the modulus related to the relaxation processes under study.

A similar sample but with a much smaller grain size has been obtained by adding 2 at.\% Ti to the NiMnSn alloy 
Table 1. Values of relevant parameters involved in the measurement procedure of the new device for the case of niobium samples at the Snoek peak temperature $\left(T_{\text {peak }}\right)$ and RT.

\begin{tabular}{|c|c|c|c|c|c|c|c|c|c|c|c|c|c|}
\hline $\begin{array}{l}\mathrm{Nb} \\
\text { sample }\end{array}$ & Temperature & $Q_{\mathrm{T}}^{-1} \times 1$ & $0^{5} W_{\mathrm{T}}(\mu \mathrm{J})$ & $\begin{array}{l}Q_{\mathrm{sb}}^{-1} \\
\left(\times 10^{5}\right)\end{array}$ & $\begin{array}{l}W_{\mathrm{sb} 1} \\
(\mu \mathrm{J})\end{array}$ & $\begin{array}{l}W_{\mathrm{sb} 2} \\
(\mu \mathrm{J})\end{array}$ & $\begin{array}{l}E_{\mathrm{sb}} \\
(\mathrm{GPa})\end{array}$ & $\begin{array}{l}Q_{\mathrm{CG}}^{-1} \\
\left(\times 10^{5}\right)\end{array}$ & $\begin{array}{l}W_{\mathrm{CG}} \\
(\mu \mathrm{J})\end{array}$ & $E_{\mathrm{CG}}(\mathrm{GPa})$ & $\begin{array}{l}Q_{\mathrm{Nb}}^{-1} \\
\left(\times 10^{4}\right)\end{array}$ & $\begin{array}{l}W_{\mathrm{Nb}} \\
(\mu \mathrm{J})\end{array}$ & $\begin{array}{l}E_{\mathrm{Nb}} \\
(\mathrm{GPa})\end{array}$ \\
\hline \multirow[t]{2}{*}{$\mathrm{Nb} 1$} & RT & 8.12 & 38.84 & 7.7 & 17.23 & 17.89 & 71.7 & 5.5 & 2.01 & 84.0 & 1.98 & 1.71 & 103.3 \\
\hline & $T_{\text {peak }}$ & 18.70 & 38.84 & 7.8 & 17.23 & 17.89 & 73.4 & 5.5 & 2.01 & 84.0 (RT) & 25.8 & 1.71 & 102.6 \\
\hline \multirow[t]{2}{*}{$\mathrm{Nb} 2$} & $\mathrm{RT}$ & 7.84 & 49.01 & 7.7 & 22.48 & 24.09 & 71.7 & 5.5 & 1.99 & 84.0 & 3.25 & 0.45 & 103.6 \\
\hline & $T_{\text {peak }}$ & 9.66 & 49.01 & 7.8 & 22.48 & 24.09 & 73.4 & 5.5 & 1.99 & 84.0 (RT) & 22.1 & 0.45 & 102.7 \\
\hline
\end{tabular}

Note: $Q^{-1}$ : damping; $W$ : storage energy; $E$ : the Young modulus. The sub-indexes sb1, sb2, CG, Nb1 and Nb2 correspond to spacer bar 1 , spacer bar 2 , crystal gauge, niobium sample 1 and niobium sample 2, respectively.

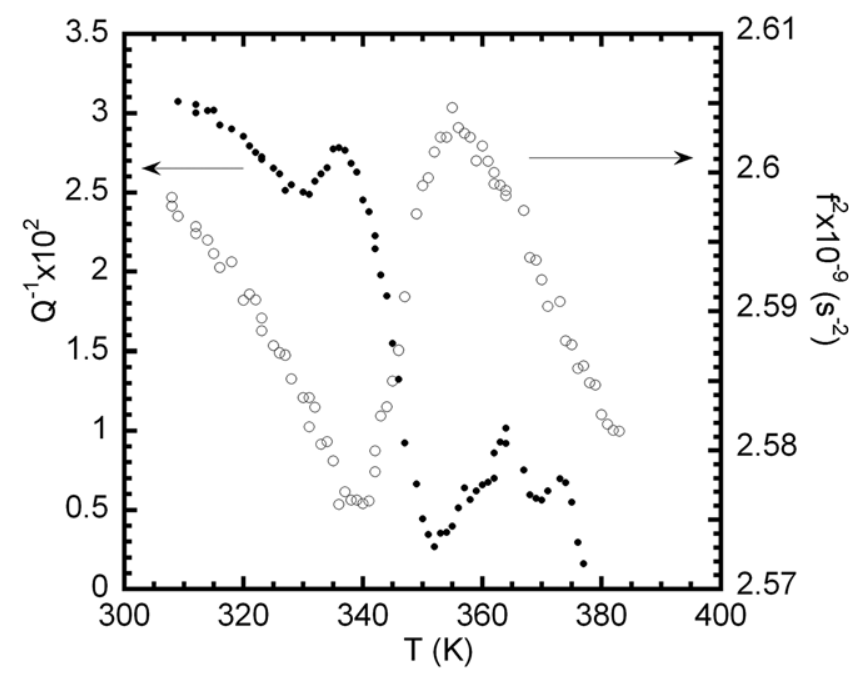

Figure 7. Damping and resonant frequency as a function of temperature for a ferromagnetic shape memory alloy (Ni-Mn-Sn), $\mathrm{Ti}=0$, around the martensitic transformation (MT).

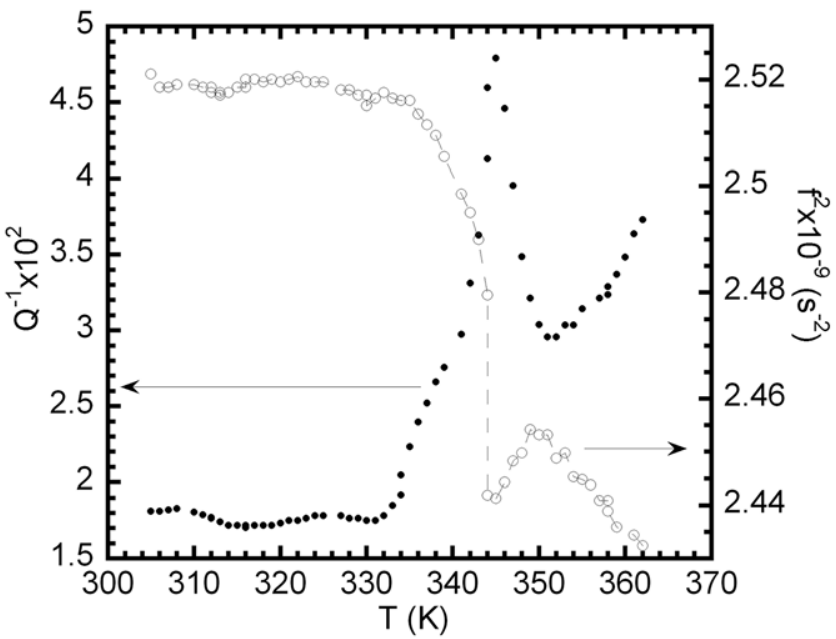

Figure 8. Damping and resonant frequency as a function of temperature for a ferromagnetic shape memory alloy (Ni-Mn-Sn), $\mathrm{Ti}=2$, around the martensitic transformation (MT). The dashed line is a guide for the eye.

$(\mathrm{Ti}=2$ sample). Figure 8 shows the corresponding behaviour of damping and frequency. Again, the change in the damping peak and the square-frequency reveals that the martensitic transition is now around $345 \mathrm{~K}$. In addition, different relaxation processes around the MT were also detected as a hump in the low temperature tail of the martensitic peak or by the two-stage modulus change. In both cases, the temperature of the martensitic transition agrees with differential scanning calorimetry (DSC) measurements.

It is convenient to mention here that the error in damping values measured for FSMA samples $\left(10^{-2}\right)$, even when their lengths are similar to $\mathrm{Nb} 2$, is smaller than $15 \%$. Indeed, the storage energy in weighted equation (32) for FSMA samples, is one order of magnitude larger than for $\mathrm{Nb} 2$ sample, due to their larger sections.

\section{Conclusions}

A novel piezoelectric device for measuring mechanical spectroscopy as a function of temperature and strain in small samples with lengths that do no match in half wavelength has been demonstrated. It is composed of a thin-flat plate quartz crystal driver, two spacer bars, the sample and the quartz crystal gauge. The driver is working outside of its resonance frequency, the spacer bars and sample do not match in frequency, and the crystal gauge is cut in half wavelength. The equipment operates outside of the tuning condition and in free decaying oscillations.

The mathematical equations related to the new equipment and the procedure for measuring mechanical spectroscopy have been given.

Measurements were performed successfully in polycrystalline niobium, to check the good functioning of the equipment, and also in small and brittle samples of $\mathrm{Ni}-\mathrm{Mn}-\mathrm{Sn}$ ferromagnetic shape memory alloys in order to determine the martensitic transition temperature. Indeed, it should be emphasized that this equipment is an important solution for measuring small and brittle samples that cannot be matched in frequency.

\section{Acknowledgments}

This work has been carried out with the financial support of the CONICET-PIP 2098 and 0179, and the PID-UNR; ING 453 (2014-2017). The authors wish to acknowledge the Cooperation Agreement between the Universidad Pública de Navarra and the Universidad Nacional de Rosario, Res. 5789/2013, and Universidad del País Vasco and the Universidad Nacional de Rosario, Res. CS.788/89-1792/2003, UPV224.31014553/02, Res. 3469/2007, Res. CS. 124/2010. 


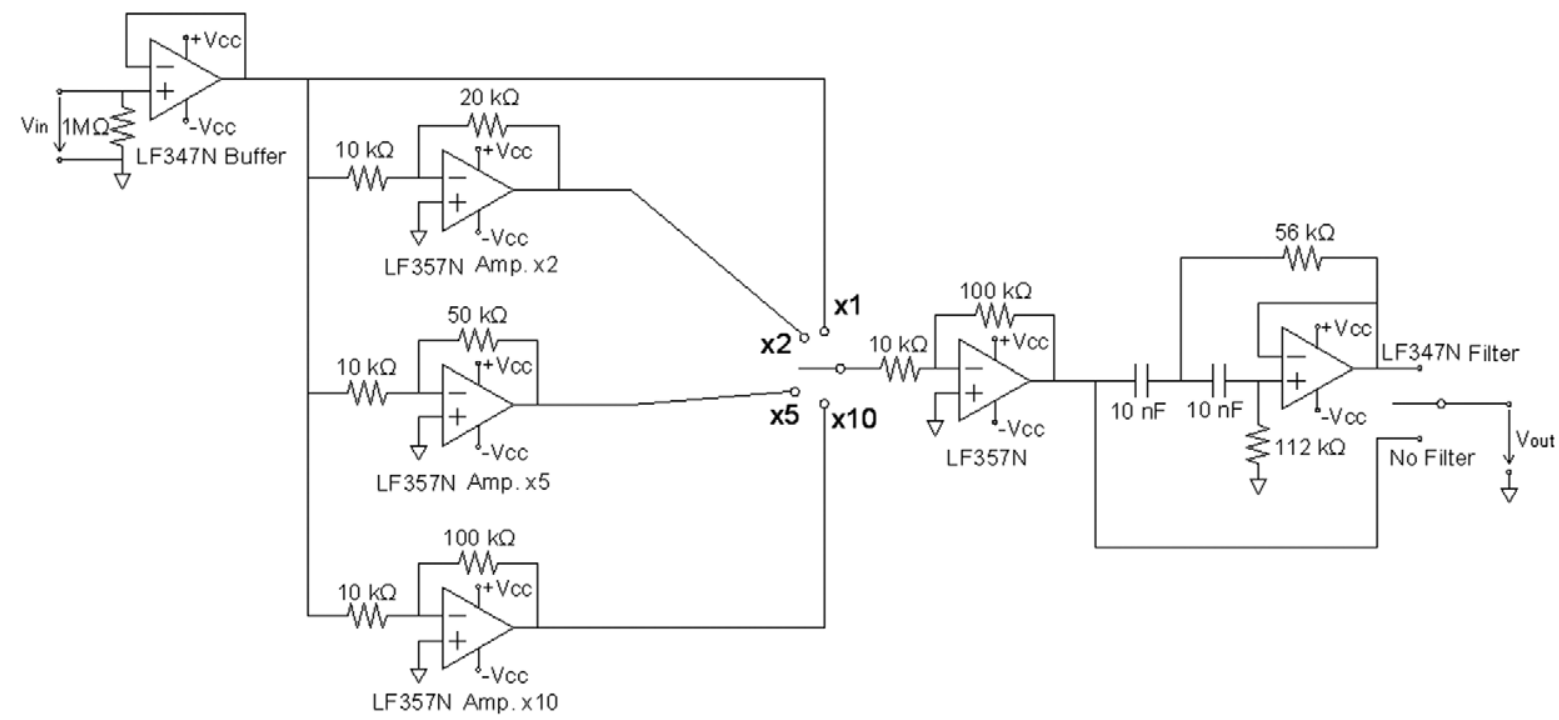

Figure A1. Electrical circuit for the high frequency amplifier with variable output.

\section{Appendix}

The amplifier is composed of a buffer, a second stage involving three operational amplifiers $\times 2, \times 5$ and $\times 10$, a third stage with an output $\times 10$ and a final stage being an active low pass filter with cut frequency at $50 \mathrm{~Hz}$. Figure A1 shows the electrical circuit for the high frequency amplifier.

\section{References}

[1] Schaller R, Fantozzi G and Gremaud G (ed) 2001 Mechanical Spectroscopy (Switzerland: Trans Tech Publ. Ltd)

[2] Nowick A S and Berry B S 1972 Anelastic Relaxation in Crystalline Solids (New York: Academic)

[3] Mason W P 1958 Physical Acoustic and the Properties of solids (New York: Van Nostrand-Reinhold)

[4] Quimby S L 1925 On the experimental determination of the viscosity of vibrating solids Phys. Rev. 25 558-73

[5] Balamuth L 1934 A new method for measuring elastic moduli and the variation with temperature of the principal Young's modulus of rocksalt between $78 \mathrm{~K}$ and $273 \mathrm{~K}$ Phys. Rev. $45715-720$

[6] Cooke W T 1936 The variation of the internal friction and elastic constants with magnetization in iron, part I Phys. Rev. 50 1158-64

[7] Michener J W and Handloser J 1947 Apparatus for Measuring Young's Modulus and Decrement of Graphite and Metals Bulletin MDDC 1428 (Desclassified: United State Atomic Energy Commission)

[8] Marx J 1951 Use of the piezoelectric gauge for internal friction measurements Rev. Sci. Instrum. 22 503-9

[9] Niblett D H and Wilks J 1960 Dislocation damping in metals Adv. Phys. 9 1-88

[10] Robinson W and Edgar A 1974 The piezoelectric method of determining mechanical damping at frequencies of 30 to $200 \mathrm{KHz}$ IEEE Trans. Sonics Ultrason. 21 98-105

[11] Harmouche M R and Wolfender A 1985 Modulus measurements in ordered $\mathrm{Co}-\mathrm{Al}, \mathrm{Fe}-\mathrm{Al}$ and $\mathrm{Ni}-\mathrm{Al}$ alloys J. Test. Eval. 13 424-8

[12] Povolo F, Marzocca A J and Goyanes S N 1992 A high frequency resonant method for the determination of the dynamic mechanical properties of solid polymers Polymer 33 2709-14

[13] Marx J W and Sivertsen J M 1953 Temperature dependence of the elastic moduli and internal friction of silica and glass J. Appl. Phys. 24 81-7

[14] Devine S D, Robinson W H and Collins M A 1974 Effect of strain on the ESR parameters for tetragonal $\mathrm{Gd}^{3+}$ in $\mathrm{CaF}_{2}$ using ultrasonic modulation J. Magn. Reson. 13 1-10

[15] Robinson W H, Collins M A and Devine S D 1975 The ultrasonic modulation technique of determining strain effects in ESR J. Phys. E: Sci. Instrum. 8 139-43

[16] Devine S D and Robinson W H 1977 Piezoelectric method of determining mechanical properties of a small sandwich specimen at 30-200 kHz J. Appl. Phys. 48 1437-41

[17] Planes A, Mañosa L and Acet M 2009 Magnetocaloric effect and its relation to shape-memory properties in ferromagnetic Heusler alloys J. Phys.: Condens. Matter 21233201

[18] Van Humbeeck J 1985 High damping capacity due to microstructural interfaces Proc. of an Int. Symp. held in Conjunction with ASM'2 Materials Week and TMS/AIME Fall Meeting (Toronto, 1985)

[19] Van Humbeeck J 1996 Damping properties of shape memory alloys during phase transformation $J$. Physique IV JP $6371-80$

[20] Pérez-Landazábal J I, Lambri O A, Bonifacich F G, SánchezAlarcos V, Recarte V and Tarditti F 2015 Influence of defects on the irreversible phase transition in Fe-Pd ferromagnetic shape memory alloys Acta Mater. 86 110-7

[21] Caddy W G 1946 Piezoelectricity (New York: McGraw-Hill)

[22] Lazan B J 1968 Damping of Materials and Members in Structural Mechanics (London: Pergamon)

[23] Lambri O A 2000 A review on the problem of measuring nonlinear damping and the obtainment of intrinsic damping Materials Instabilities ed J Martinez-Mardones et al (New York: World Scientific)

[24] Lambri O A 1994 New procedure for determining internal friction parameters of tension-induced relaxation processes with distribution of relaxation times Mater. Trans. JIM 35 458-65

[25] Weller M, Li G Y, Zhang J X, Ke T S and Diehl J 1981 Accurate determination of activation enthalpies associated with the stress-induced migration of oxygen or nitrogen in tantalum and niobium Acta Metall. 29 1047-54 
[26] Weller M, Zhang J X, Li G Y, Ke T S and Diehl J 1981 Internal friction study on the existence of oxygen pairs in interstitial solid solution of tantalum with oxygen Acta Metall. 29 1055-60

[27] Molinas B J, Lambri O A and Weller M 1994 Study of nonlinear effects related to the Snoek-Köster relaxation in $\mathrm{Nb}$ J. Alloys Compd. 211-212 181-4

[28] Zelada-Lambri G I, Lambri O A and García J A 2006 Mechanical energy losses due to the movement of dislocations in molybdenum at high temperatures $(0.3 \mathrm{Tm})$ J. Nucl. Mater. 353 127-34
[29] Molinas B J and Povolo F 1987 Analysis of the problem of converting measured amplitude-dependent internal friction to intrinsic values. Detailed study of the case of forced oscillations J. Phys. E: Sci. Instrum. 20 970-6

[30] Schulze K, Grallath E and Weller M 1981 Determination of small oxygen contents in niobium by measurement of electrical resistivity and internal friction Z. Metallkd. 72 439-44

[31] Weller M, Zhang J X, Schulze K, Ke T S and Diehl J 1981 The Snoek relaxation in tantalum and niobium with relatively high concentrations of oxygen J. Phys. Colloq. 42 817-22 